Reprod. Nutr. Dévelop., 1986, 26 (1 B), 323-324.

\title{
Enregistrement chronique des variations du débit duodénal chez les bovins
}

\author{
Y. RUCKEBUSCH, A. MERRITT, C. PONCET(*)
}

Ecole Nationale Vétérinaire, Laboratoire de Physiologie, 31076 Toulouse.

(*) Laboratoire de la Digestion des Ruminants,

I.N.R.A. Theix, 63122 Ceyrat, France.

Summary. Estimates of the digesta flow through the duodenum were measured using an electromagnetic flow-meter in steers fitted with re-entrant cannulae. The mean net aboral flow ranged from 2700 to $3600 \mathrm{ml} / \mathrm{h}$ over a period of 5 months after surgery. The flow pattern showed periods of peristaltic and antiperistaltic movements separated by periods of quiescence related to the basic rest-activity cycle of the small intestine.

La débitmétrie électromagnétique autorise la mesure directe du flux duodénal pendant de longues périodes chez le mouton ou la chèvre (Singleton, 1961) et chez le veau (Dardillat, 1977). La technique utilisée fait appel à la mise en place d'une canule réentrante avec transection de I'intestin 5 à $10 \mathrm{~cm}$ en aval du pylore (Poncet et al., 1977). On peut également envisager de placer le débitmètre autour de la paroi (Dardillat, 1977) ou de l'insérer dans des canules en $Y$ (Poncet et Ivan, 1984), ce qui évite la transection duodénale. Or, exception faite de la présence d'une simple canule en $\mathrm{T}$, l'activité motrice cyclique de la zone gastro-duodénale est toujours fortement accrue pendant une période postopératoire d'environ 3 à 4 semaines chez le mouton. La présente étude concerne l'intérêt de la débitmétrie extracorporelle avec transection duodénale chez les bovins.

Matériel et méthodes. Deux bouvillons sevrés à l'âge de 4 mois et pesant 90 $110 \mathrm{~kg}$ ont été munis 3 et 6 semaines après sevrage d'une canule réentrante (diam. int. $15 \mathrm{~mm}$ ) après section transversale du duodénum effectuée à $10-12 \mathrm{~cm}$ du pylore. Des électrodes placées en amont et en aval de la canule réentrante permettent de recueillir l'électromyogramme et partant, le cycle moteur de l'intestin grêle (Ruckebusch et Bardon, 1984). La mesure du flux duodénal est effectuée en plaçant une sonde de débitmétrie électromagnétique (Poncet et al., 1977) entre les deux extrémités de la canule à partir du $3^{\mathrm{e}}$ jour après l'intervention. L'extrémité d'une électrode à $\mathrm{pH}$ (diam. $2 \mathrm{~mm}$ ) est placée en amont de la sonde débitmétrique.

Les enregistrements ont été effectués de façon continue à l'aide d'un polygraphe pour des périodes allant de $12 \mathrm{~h}$ à $48 \mathrm{~h}$ et à raison de 1 à 2 séances par semaine durant 5 mois chez les deux animaux recevant à volonté du foin de graminées renouvelé à $8 \mathrm{~h}$ et $16 \mathrm{~h}$. Des contrôles pendant 30 à $60 \mathrm{~min}$ du volume de chyme évacué de 5 en 5 min par l'extrémité orale de la canule, avec ou sans réintroduction du contenu digestif, ont été effectués vers 9 et $15 \mathrm{~h}$. 
Résultats et discussion. Les deux animaux ont présenté une activité motrice cyclique de période comprise entre 40-50 min et sans évolution significative en phase postopératoire ( $\mathrm{du} 3^{\mathrm{e}}$ au $24^{\mathrm{e}}$ jour) et pendant les 5 mois d'observation. La figure 1 montre que les passages de contenu digestif ne sont ni équidistants, ni isodynames durant des périodes d'activité de 40-50 min. Celles-ci sont séparées par des périodes de repos de 5-8 min. Le débit duodénal est caractérisé par l'intermittence des flux et reflux correspondant à la propulsion aborale nette variant de $2700 \mathrm{ml} / \mathrm{h}$ à $3600 \mathrm{ml} / \mathrm{h}$ en période interdigestive. Le débit atteint 4700 $5500 \mathrm{ml} / \mathrm{h}$ pendant l'heure qui suit la distribution de foin. Un même ordre de grandeur a été observé pour des mesures directes du chyme pendant $30 \mathrm{~min}$, qu'il y ait ou non réintroduction des digesta en aval. Le $\mathrm{pH}$ du contenu duodénal oscille entre 3 et 4,5 avec des pics atteignant 5,5 au début de chaque passage aboral de contenu duodénal. II varie peu durant les reflux et les périodes de repos. Les variations suivantes de la motricité gastroduodénale ont été provoquées: récurrence accrue des complexes myoélectriques par l'administration de méthysergide $(0,05 \mathrm{mg} / \mathrm{kg} \mathrm{SC})$ (Ruckebusch et Bardon, 1984); inhibition de l'évacuation gastroduodénale par la xylazine $(0,1 \mathrm{mg} / \mathrm{kg}$ IM) ou la morphine $(1,25 \mathrm{mg} / \mathrm{kg}$ IV). L'augmentation synchrone des périodes de débit et des cycles moteurs a été la règle après le méthysergide. Tout transfert aboral du chyme était supprimé pendant 2 à $3 \mathrm{~h}$ après la xylazine. Le débit net était également nul pendant $2 \mathrm{~h}$ après la morphine, mais dans ce cas, en raison d'une importance égale des flux et reflux au niveau duodénal.

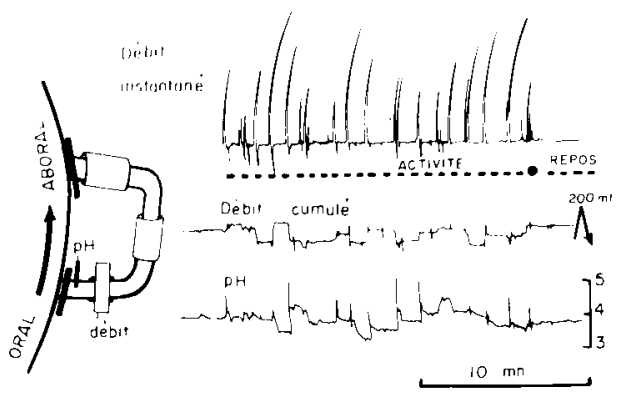

FIG. 1. - Exemple d'enregistrement des variations de passage du chyme gastrique, débit instantané et débit cumulé, chez l'un des bouvillons à l'âge de 7 mois $(160 \mathrm{~kg})$. L'intermittence des périodes de débit correspond à la périodicité des complexes myoélectriques avec absence de débit et stabilité du $\mathrm{pH}$ durant les phases de repos.

Au total, la mise en place d'une canule réentrante en vue de l'utilisation de la débitmétrie extracorporelle ne modifie pas de façon majeure l'activité cyclique de l'intestin, même en période postopératoire chez les jeunes bovins. Par ailleurs, le procédé, s'il atténue la vitesse réelle des mouvements liquides, permet néanmoins de différencier de façon satisfaisante la réduction de débit liée à l'absence de propulsion de celle due à une augmentation des reflux duodéno-gastriques.

Dardillat C., 1977. Analyse électromyographique et débitmétrique du transit alimentaire chez le veau nouveau-né. J. Physiol., Paris, 73, 925-944.

Poncet C., Dimova E., Léveillé M., Dardillat C., 1977. Mise au point d'une méthode d'enregistrement chronique du débit duodénal chez le mouton. Ann. Biol. anim. Bioch. Biophys., 17, 515-522.

Poncet C., Ivan M., 1984. Effect of duodenal cannulation in sheep on the pattern of gastrointestinal motility and digestive flow Reprod. Nutr. Dévelop., 24, 887-902.

Ruckebusch Y., Bardon Th., 1984. Le cycle moteur de l'intestin grêle. C. R. Acad. Sci., 298. 403-408.

Singleton A. G., 1961. The electromagnetic measurement of the flow of digesta through the duodenum of the goat and sheep. J. Physiol., Lond., 155, 134-147. 